\title{
Non-Association of Toll-Like Receptor 2 rs3804099 Polymorphism with Paediatric Tuberculosis in South Africa
}

\author{
Inyang U. Udosen \\ Department of Biological Sciences, Akwa Ibom State University, Mkpat-Enin, AKS, Nigeria
}

\begin{abstract}
Genetic polymorphisms are important risk factors that are involved in development and severity of disease infections especially tuberculosis. In this study, a non-synonymous single nucleotide polymorphism of immune response protein gene, toll-like receptor 2 (TLR2) occurring in exon 2 (rs3804099) was carried out by genotypic analysis of cases and controls for tuberculosis. The paediatric study population consisted of 151 cases and 82 controls obtained mainly from Xhosa ethnic group.Result from this study showed that this non-synonymous single nucleotide polymorphism of TLR2 was not associated with tuberculosis $(\mathrm{p}=0.38)$.
\end{abstract}

Keywords: Tuberculosis, Toll-like Receptor 2, Paediatrics, Single Nucleotide Polymorphisms

DOI: $10.7176 /$ ALST/74-06

Publication date:May $31^{\text {st }} 2019$

\subsection{INTRODUCTION}

Toll-like receptors are type 1 transmembrane proteins which detect microbes that have breached the physical barriers such as skin or intestinal tract mucosa and activate immune cell responses. They play a major role in the innate immune system. Toll-like receptors are a type of pattern recognition receptors which recognize carbohydrate molecules on the surfaces of pathogens collectively referred to as pathogen-associated molecular patterns (PAMPS). Nomura and co-workers discovered the human toll-like receptors in 1994 and which were mapped to a chromosome by Taguchi and colleagues in 1996. Toll-like receptors were named due to their similarity to Toll, a receptor identified in 1996 by Lemaitre and his colleagues in the fruit fly Drosophila melanogaster for their role in the fly's immunity to fungal infection achieved via the synthesis of antimicrobial peptides.

Takeda and colleagues (2003) reported that the signaling pathway of Drosophila Toll has similarity with mammalian IL-1 pathway that causes activation of NF- $\mathrm{kB}$, a transcription responsible for many reactions of inflammatory and immune responses. They suggested that the toll pathway in drosophila is necessary for resistance to Gram-positive bacterial infections in addition to fungal infections. The TLR2 gene consist of two exons but all the coding sequence is in exon 2 .

The expression of TLR2 is regulated by many factors such as microbial invasion, microbial components and cytokines.

TLR2 can detect components from several microorganisms. They include lipoproteins from Gram-negative bacteria, Mycoplasma and Spirochaetes; peptidoglycan and lipoteichoic acid from Gram-positive bacteria; lipoarabinomannan from Mycobacteria; glycoinositolphospholipids from Trypanosoma cruzi; a phenol-soluble modulin from Staphylococcus epidermis; zymosan from fungi; glycolipids from Treponema maltophilum and porins from outer membrane of Neisseria.

A major study by Liu and colleagues (2006) has shown that TLR activation resulted in macrophage inductive expression of vitamin D receptor and vitamin D-1-hydroxylase genes to cause production of antimicrobial peptide cathelicidin and killing of intracellular Mycobacterium tuberculosis.

Mutations in the TLR2 gene has been confirmed in many studies to result in attenuated immune response (Lorentz et al., 2000; Yim et al., 2006 and Kang \& Chae, 2001). Lorentz and Co-workers (2000) demonstrated that mutation in the TLR2 gene- Arg753Gln polymorphism- showed less response to bacterial peptides derived from Borrelia burgdoferi and treponema pallidum. In addition, they reported that subjects with TLR2 Arg753Gln polymorphism had Staphylococcal infections and proposed that this polymorphism may render individuals susceptible to harmful bacterial infections especially septic shock. Lorentz and Co-workers also suggested that Carboxyl terminus of TLR2 which is involved in signaling might be affected by this polymorphism because Arg753Gln is necessary for the receptor function. However, a study by Ryu and Co-workers (2006) reported that both TLR2 Arg677Trp and Arg753Gln polymorphisms were not responsible for host susceptibility to Nontuberculous Mycobacterial lung diseases in the South Korean population but another report by Yim and Coworkers (2006) showed association of guanine-thymine repeat polymorphisms in intron2 of the TLR2 gene and the predisposition to clinical tuberculosis. Also, Kang and Chae (2001) had earlier reported the implication of TLR2 Arg677Trp polymorphism in the development of lepromatous leprosy.

Not much work has been carried out an association of TLR with tuberculosis. In one study by Ben-Ali and Co-workers (2004), TLR2 Arg677Trp polymorphism was associated with predisposing individuals to tuberculosis infection in Tunisian subjects. Another study in turkey carried out by Ogus and Co-workers (2004) 
reported the association of Arg753Gln polymorphism of the TLR2 gene to be a risk factor in the development of clinical tuberculosis.

The TLR2 polymorphism under study did not involve any amino acid change (non-synonymous SNP) and it occurred in the coding region of exon 2.

\subsection{MATERIALS AND METHODS}

\subsection{Study Population}

The samples for the population study which consisted of cases and controls were obtained from the region with the highest tuberculosis incidence in South Africa which is Capetown in Western Cape Province.

The study subjects and samples used for the study were 233 individuals consisting of 151 paediatric patients and 82 controls. The ages of paediatric cases and controls ranged from 6 months to 14 years having median ages of 69 months and 74 months for the paediatric cases and controls respectively. They were obtained from two ethnic groups in South Africa: Xhosa and Coloureds. The study population were made up of 198 Xhosa and 33 Coloureds. Patients recruited for the study were diagnosed as having had tuberculosis, some were past history of the disease. The diagnostic criterion for the presence of tuberculosis was the use of radiological test to confirm findings associated with the disease, reactivity test or Mantoux test for M. tuberculosis together with pathological findings of tuberculosis disease in lymph node, lungs e.t.c. Genomic DNA used for genotyping was isolated from whole blood using QIAGEN DNA purification kit. The genomic DNA of these cases and control study samples were the original source of DNA.

Patients who were HIV positive were excluded as well as those that did not have definitive evidence for the disease. The control group consisted of unrelated subjects that had been diagnosed as not having any history of the disease. The data for all the paediatric patients and paediatric controls including sex and ethnic groups were collated from their medical records for this study.

The cohorts for this study were recruited from different areas of Capetown. The cases were obtained from Red Cross Children's Hospital in capetown and controls were contacts of the cases.

The Xhosa ethnic group used in this study is the second largest ethnic group of the Black South Africans. The Coloured ethnic group are a distinct population that could be considered as mixed population that are different from Black, White or Asian. This study group included one Caucasian as well as an Indian case.

Samples were obtained in accordance with guidelines and approval from Ethics Committee of the Red Cross Children's Hospital.

\subsection{TLR2 Genotyping}

For TLR2 SNP rs3804099, genotyping was performed by ARMS PCR (Newton et al., 1989) using 5'AAAGTTTGAAGTCAATTCAGAAT3' and 5' AAAGTTTGAAGTCAATTCAGAAC3' as forward primers for T and $\mathrm{C}$ allele respectively with 5'ATAAGTAAAACCTTGGAATAT3' as reverse primer. The PCR was performed in $25 \mu \mathrm{l}$ under the following conditions: 2 minutes of denaturation at $94^{\circ} \mathrm{C}$ followed by 30 cycles of denaturation for 30 seconds at $94^{\circ} \mathrm{C}$, annealing of primers to the template for 30 seconds at $53^{\circ} \mathrm{C}$ and extension at $72{ }^{\circ} \mathrm{C}$ for 30 seconds. A final extension was carried out at $72{ }^{\circ} \mathrm{C}$ for 5 minutes. PCR products obtained were separated by subjecting them to electrophoresis in a $2 \%$ agarose gels and visualized under UV fluorescence for identification of the bands.

\subsection{RESULTS}

The SNP in the TLR2 gene- rs3804099 was genotyped using ARMS PCR to generate 399-base pair fragment (figure 1)

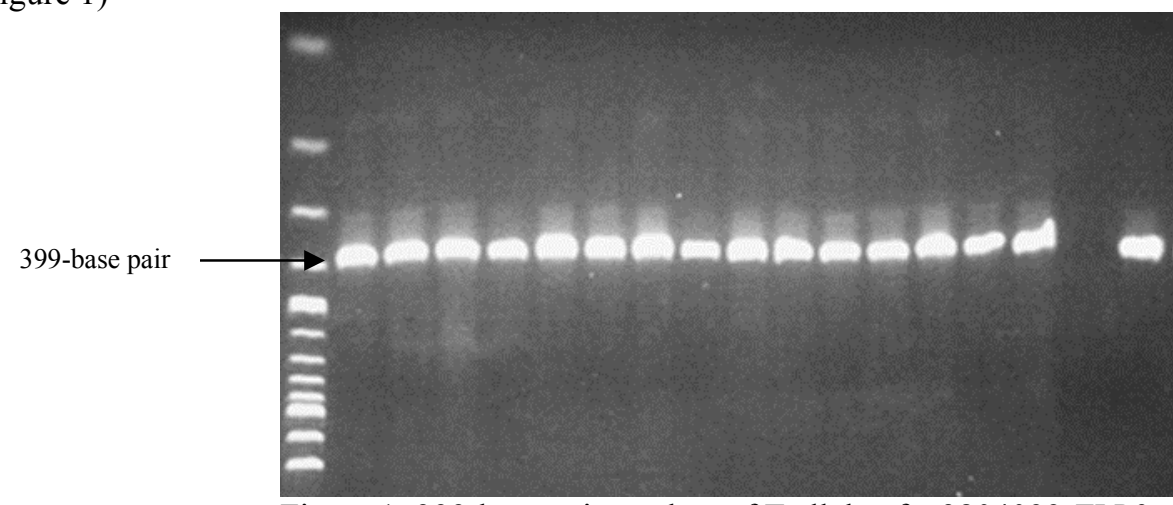

Figure 1: 399-base pair product of T allele of rs3804099 TLR2

The result of genotypic screening study showed a predominance of heterozygotes over homozygotes (table 1). The heterozygote accounted for $94 \%$ genotype frequency in cases compared with $91 \%$ in controls. 
Analysis of the study population for rs3804099 showed it was not significant $(\chi 2=1.917$ at $\mathrm{p}=0.38)$.

Table 1: Genotype frequency of rs3804099 TLR2 in the study population

\begin{tabular}{|l|l|l|l|}
\hline & TT & CT & CC \\
\hline Cases & $7(5 \%)$ & $140(94 \%)$ & $1(1 \%)$ \\
\hline Controls & $7(9 \%)$ & $76(91 \%)$ & $0(0 \%)$ \\
\hline
\end{tabular}

The allele frequency of rs3804099 TLR2 in the study population was $142(48 \%)$ for $\mathrm{C}$ allele and $154(52 \%)$ for $\mathrm{T}$ allele in paediatric cases; controls had allele frequency of $74(46 \%)$ and $88(54 \%)$ for $\mathrm{C}$ and $\mathrm{T}$ allele respectively. Comparison of allele frequency of cases and controls also gave no significant difference $(\chi 2=0.221$ at $\mathrm{p}=0.65$ ).

The coloured population had the frequency of 3, 17, 0 for TT, CT and CC genotype respectively in cases while its controls had 2, 8 and 0 for TT, CT and CC genotypes.

In the Xhosa population, the occurrence of TT, CT and CC genotype in cases were 3, 118 and 1 respectively. The controls were found to have 5, 66 and 0 for TT, CT and CC genotypes. Analysis of the Xhosa paediatric study population genotype showed it was statistically non-significant $(\chi 2=2.923$ at $p=0.23)$. The rs3804099 TLR2 allele frequency in Xhosa paediatrics for $\mathrm{C}$ and T allele were 120 and 124 respectively in cases while those of paediatric controls were 61 and 79 respectively. Statistical analysis of Xhosa cases and controls' allele frequencies gave no significant difference $(\chi 2=1.123$ at $\mathrm{p}=0.29)$.

The gender study population showed a genotype distribution suggesting a predominance of heterozygote (table 2).

Table 2: Genotype distribution of rs3804099 TLR2 in gender cases study population

\begin{tabular}{|l|l|l|l|}
\hline & TT & CT & CC \\
\hline Males & $4(6.7 \%)$ & $56(93.3 \%)$ & $0(0 \%)$ \\
\hline Females & $2(2.4 \%)$ & $82(96.5 \%)$ & $1(1.1 \%)$ \\
\hline
\end{tabular}

Analysis of the study population showed it was non-significant $(\chi 2=2.324$ at $p=0.31)$. The allele frequency of the gender study population for $\mathrm{C}$ and $\mathrm{T}$ allele were 56 and 64 respectively in males as well as having 84 and 86 respectively in females. Comparison of the allele frequencies of the male and females of the study population showed no statistically significant difference $(\chi 2=0.212$ at $\mathrm{p}=0.65)$.

The Xhosa male cases showed the genotype frequency of 2, 47 and 0 for TT, CT and CC genotypes respectively as compared to its female cases of 1, 71 and 1 for TT, CT and CC genotypes respectively. The genotype distribution between the male and female cases in Xhosa population showed no significant difference $(\chi 2=1.553$ at $p=0.46)$. Xhosa geneder population showed a higher frequency of $\mathrm{T}$ allele. The Xhosa paediatric males had allele frequencies of 47 and 51 for $\mathrm{C}$ and $\mathrm{T}$ allele respectively while females had the same allele frequency of 73 for both $\mathrm{C}$ and $\mathrm{T}$ allele. Comparison of allele frequency between Xhosa gender also gave no significant difference $(\chi 2=0.098$ at $\mathrm{p}=0.75)$. The paediatric population had allele frequencies of 70 and 80 for $\mathrm{C}$ and $\mathrm{T}$ allele respectively in extra-pulmonary tuberculosis while pulmonary tuberculosis had 67 for $\mathrm{C}$ allele and 73 for $\mathrm{T}$ allele. Statistical analysis of its allele frequencies was not significant $(\chi 2=0.041$ at $\mathrm{p}=0.84)$.

The occurrence of genotypes in the type of tuberculosis in the study population (table 3 ) also showed no significant difference between extra-pulmonary and pulmonary tuberculosis $(\chi 2=1.837$ at $\mathrm{p}=0.40)$.

Table 3: Genotype frequency of rs3804099 TLR2 in tuberculosis type of the study population

\begin{tabular}{|l|l|l|l|}
\hline TB Type & TT & CT & CC \\
\hline Extra-pulmonary & $6(88.2 \%)$ & $68(90.7 \%)$ & $1(1.3 \%)$ \\
\hline Pulmonary & $3(4.3 \%)$ & $67(95.7 \%)$ & $0(0.0)$ \\
\hline
\end{tabular}

Xhosa population also showed similar distribution of genotypes with the heterozygote at a higher frequency. The genotype frequencies were 0,39 and 1 for TT, CT and CC respectively in extra-pulmonary tuberculosis while pulmonary tuberculosis had 3,67 and 0 for TT, CT and CC genotype respectively. Statistical analysis between pulmonary and extra-pulmonary tuberculosis showed it was not significant $(\chi 2=3.473$ at $\mathrm{p}=0.81)$. Tuberculosis type occurrence of alleles in Xhosa paediatrics showed that allele frequency for $\mathrm{C}$ was 41 and $\mathrm{T}$ allele was 39 in extra-pulmonary tuberculosis with pulmonary cases having allele frequency of 67 and 73 for $\mathrm{C}$ and $\mathrm{T}$ alleles. Statistical analysis of pulmonary and extra-pulmonary tuberculosis allele frequency gave no significant association $(\chi 2=0.234$ at $\mathrm{p}=0.63)$.

The Coloured population had 3 and 17 for TT and CT genotypes as there was no CC genotypes while there was no case of pulmonary tuberculosis for this population.

However, the rs3804099 genotype occurrence in the study population and sub-groups was not in agreement with Hardy-Weinberg equilibrium $(\mathrm{p}=0)$.

\subsection{DISCUSSION}

In the study of rs3804099 polymorphism of TLR2, it was observed that they had no association with the tuberculosis disease in this study population and even within ethnic groups. No significant difference was found 
neither in the gender population nor Xhosa ethnic group as well as between pulmonary and extra-pulmonary tuberculosis both in its genotype distribution as well as its allelic distribution. The rs3804099 C/T polymorphism was found in 148 out of 151 cases and in 81 out of 82 controls. This polymorphism in the study population showed a genotype distribution having a predominance of heterozygotes across all groups studied.

In addition, the genotype distribution of rs3804099 TLR2 polymorphism in the control paediatrics used in this study was outside Hardy-Weinberg equilibrium. The various factors that could be responsible for this deviation are population sample consisting of subpopulation that do not completely interbreed, selection pressure in the population study in favour of heterozygote genotype as well as environmental factors which could be a direct effect of geographical location as well as small population size of the study. Indeed, environmental factors have been implicated in M. tuberculosis diversity (Brudey et al., 2006) which could exert selective pressure on the genotype of the population. Environmental factor in addition to pathogen influence could be a contributing factor in selecting the predominance of heterozygote alleles in this population study (Bellamy, 1998).

The rs3804099 TLR2 polymorphism in this population study contradicts studies of other TLR2 polymorphisms carried out in other populations (Ben-Ali et al., 2004; Kang \& Chae, 2001 and Kang et al, 2002). This study suggests that rs3804099 TLR2 polymorphism is not a contributing factor in clinical tuberculosis in this particular population. It differs from the previous study on codon 34 variant of $M B L 2$ carried out on the same population sample which showed predisposition effect to tuberculosis (Udosen \&Nya, 2017). It could be that in the present study, other polymorphisms could be a risk factor in the development of tuberculosis with the exception of the one under study. This makes a case for further investigation for the role of other polymorphisms in susceptibility of tuberculosis in this study population. The TLR2 polymorphism under study did not involve amino acid change and it is in the coding region of exon two hence it is a non-synonymous single nucleotide polymorphism.

\section{ACKNOWLEDGEMENTS}

The author acknowledges the contribution of Dr. Jon Goulding and Prof. John Armour for his contributions to the research undertaken at University of Nottingham, England. This research was sponsored by Akwa Ibom State University Fellowship.

\section{REFERENCES}

Bellamy, R. 1998, Genetic susceptibility to tuberculosis in human populations, Thorax 53:588-593.

Ben-Ali, M., Barbouche, M., Bousnina, S., Chabbou, A., and Dellagi, K. 2004, Toll-Like Receptor 2 Arg677Trp Polymorphism Is Associated with Susceptibility to Tuberculosis in Tunisian Patients, Clinical and Diagnostic Laboratory Immunology, 11(3): 625-626.

Brudey, K., Driscoll, Rigouts, L., Prodinger, W. M., Gori, A., Al-Hajoj, S. A. and Allix, C. et al. 2006, Mycobacterium tuberculosis complex genetic diversity: mining the fourth international spoligotyping database (SpolDB4) for classification, population genetics and epidemiology. BMC Microbiology 6:23.

Kang, T. J. and Chae, G. T. 2001, Detection of Toll-like receptor 2 (TLR2) mutation in the lepromatous leprosy patients. FEMS Immunol Med. Microbiology 31:53-58.

Kang, T. J., Lee, S. B. and Chae, G. T. 2002, A polymorphism in the toll-like receptor 2 is associated with IL-12 production from monocyte in lepromatous 1 leprosy. Cytokine 20:56-62.

Lemaitre, B., Nicholas, E., Michaut, L., Reichhart, J. M. and Hoffman, J. A. 1996, The dorsoventral regulatory gene cassette spatzle/Toll/cactus controls the potent antifungal response in Drosophila adults. Cell 86: 973983.

Liu, P. T., Stenger, S., Li, H., Wenzel, L., Tan, B. H., Krutzik, S. R., Ochoa, M. T., Schauber, J., Wu, K., Meinken, C., Kamen, D. L., Wagner, M., Bals, R., Steinmeyer, A., Zugel, U., Gallo, R. L., Eisenberg, D., Hewison, M., Holis,B. W., Adams, J. S., Bloom, B. R. and Modlin, R. L. 2006, Toll-like Receptor Triggering of a Vitamin D-Mediated Human Antimicrobial Response. SCIENCE. 311:770-773.

Lorenz, E., Mira, J. P., Cornish, K. L., Arbour, N. C., and Schwartz, D. A. 2000, A Novel Polymorphism in the Toll-like Receptor 2 Gene and Its Potential Association with Staphylococcal Infection. Immunity and Infection. 68(11): 6398-6401.

Newton, C. R., Heptinstall, L. E., Powell, S. J., Summers, C., Kalsheker, N., Smith, J. C. and Markham, A. F. 1989, Analysis of any point mutation in DNA. The amplification refractory mutation system (ARMS) Nucleic Acids Research17(7):2503-2515.

Nomura, N., Miyajima, N., Sazuka, T., Tanaka, A., Kawarabayashi, Y., Sato, S., Nagase, T., Seki, N., Ishikawa, K. and Tabata, S. 1994, Prediction of the coding sequences of unidentified human genes. The coding sequences of 40 new genes (KIAA0001-KIAA0040) deduced by analysis of randomly sampled cDNA clones from human immature myeloid cell line KG-1. DNA Res 1:27-35.

Ogus, A. C., Yoldas, B., Ozdemir, T., Uguz, A., Olcen, S., Keser, I., Coskun, M., Cilli, A. and Yegin, O. 2004, The Arg753Gln polymorphism of the human Toll-like receptor 2 gene in tuberculosis disease. Eur Respir $J$ 
23:219-223.

Ryu, Y. J., Kim, E. J., Koh, W., Kim, H., Kwon, O. J. and Chang, J. H. 2006, Toll-like Receptor 2 Polymorphisms and Nontuberculous Mycobacterial Lung Diseases. Clinical and Vaccine Immunology. 13(7):818-819.

Taguchi, T., Mitcham, J. L., Dower, S. K., Sims, J. E. and Testa, J. R. 1996, Chromosomal localization of TIL, a gene encoding a protein related to the Drosophila transmembrane receptor Toll, to human Chromosome 4p14. Genome 32:486-488.

Takeda, K., Kaisho, T. and Akira, S. 2003, Toll-Like Receptors. Annu. Rev. Immunol. 21:335-76

Udosen, I. U. and Nya, E. J. 2017 Association of MBL2 codon 34 variant of Exon 1 with severity of tuberculosis in Paediatric Population of South Africa. Advances in Life Science and Technology, ISSN (Paper 222-7181) ISSN (Online) 2225-062X, Vol. 58

Yim, J. J., Lee, H. W., Lee, H. S., Kim, Y. W., Han, S. K., Shim, Y. S. and Holland, S. M. 2006. The association between microsatelite polymorphisms in intron II of the human Toll-like receptor 2 gene and tuberculosis among Koreans. Genes Immunol. 7(2):150-5. 